\title{
A theoretical approach to optimal association control in vehicular Wi-Fi networks
}

\author{
Jaeryong Hwang ${ }^{1}$, Jaehyuk $\mathrm{Choi}^{2}$, Joon Yoo ${ }^{2}$ and Chong-kwon Kim ${ }^{3 *}$
}

\begin{abstract}
The recent ubiquitous deployment of Wi-Fi access points (APs) has offered vehicles to use the high-speed and low-cost Internet service via the roadside APs. However, the high mobility of vehicles and the limited coverage of APs render some challenges. First, it results in frequent handoffs, thus leading to long delay and low service availability. Second, the available AP sets and their channel quality change dynamically, making the AP selection problem even harder. Therefore, there is a strong need to develop an efficient association control mechanism that provides efficient vehicular Wi-Fi access. In this paper, we present a theoretical framework to formulate the optimal association problem through non-linear integer programming, whose objective function is to maximize the throughput or to minimize the handoff overhead. We show that this problem holds the totally unimodular (TU) property and is thus solvable in polynomial time. Then, we study the optimality of association control by comparing existing online algorithms through real trace-based simulations. The results show that there exists a large performance gap between the performance of existing online algorithms and the optimal one. We also observe that the association control algorithm can be further improved if it has access to future knowledge. Particularly, the offline optimal with future AP information improves the performance of the local optimal by up to $10 \%$.
\end{abstract}

Keywords: Vehicular Wi-Fi access; Association control; Optimization

\section{Introduction}

Recently, Wi-Fi access technology for moving vehicles has drawn considerable attention, since it has been shown that Wi-Fi connectivity is feasible even at vehicular speed [1-9]. Moreover, the wide deployment of numerous WiFi access points (APs) in the urban areas [3] provides cost-effective and broadband Internet connectivity to the passengers. However, there are still several challenges remaining for vehicular Wi-Fi access. First, different from the traditional data access systems for stationary users, the connection time in vehicular Wi-Fi access is typically short, since the vehicles move fast and the coverage of roadside APs is limited. Furthermore, the available AP sets and their wireless channel change dynamically due to the high vehicle mobility. To sustain the connectivity and maintain high link quality, the vehicles have to continuously associate with different APs and thus conduct frequent handoffs. The frequent handoffs, however,

${ }^{*}$ Correspondence: ckim@snu.ac.kr

${ }^{3}$ School of Computer Science and Engineering, Seoul National University, Seoul 151-744, South Korea

Full list of author information is available at the end of the article involve very high overhead such as association, authentication, and DHCP latency $[3,6,9,10]$. Therefore, deciding when to perform the handoff and determining the best AP among the candidate APs are a very important, yet challenging, problem.

Several studies have been proposed to address the association and handoff problem. Giannoulis et al. [4] have suggested a handoff protocol which supports a vehicle's mobility in an urban mesh topology. Kim et al. [11] presented an association control solution that minimizes the frequency of handoffs of mobile devices. A recent study of Xie et al. [12] aims to improve the overall throughput and fairness for all users in an enterprise network. However, the association scheduling problem considering handoff overhead from the vehicle's point of view has not yet been addressed well despite its importance, while these existing solutions improve the association performance and provide useful insights in the design of online association algorithms.

In this paper, we present a theoretical framework for analyzing the association problem in vehicular Wi-Fi access networks, with the aim of understanding and

\section{Springer}

C 2014 Hwang et al : licensee Springer. This is an Open Access article distributed under the terms of the Creative Commons Attribution License (http://creativecommons.org/licenses/by/2.0), which permits unrestricted use, distribution, and reproduction in any medium, provided the original work is properly credited. 
improving the performance of several association control schemes. We first formulate the association problem as a non-linear integer program in which we incorporate the influence of a vehicle's mobility, available effective bit rate from APs, and handoff cost. Our key contribution is to show that the non-linear integer program can be cased into a linear programming problem thanks to the properties of the binary decision variable. Furthermore, we show that the linearized problem is solvable in polynomial time because the constraint matrix of the problem meets the totally unimodular (TU) property. The TU implies that all vertexes of the polyhedron representing the feasible set are integral; thus, the linear program obtained by relaxing the integrality constraints of the corresponding integer program can find an optimal integral solution. We prove that the optimal association problems satisfy the TU property, and we validate that these problems are solvable in polynomial time.

We conduct extensive simulations by using the metropolitan city of Seoul bus mobility trace gathered from the Seoul Transport Operation \& Information Service (TOPIS) database [13]. For the simulations, we have collected the real movement traces for more than a month of approximately 7,000 buses. We compare three existing online algorithms - bandwidth-based association, duration-based association, and combined metric-based algorithms - with the optimal solution. We have observed that there exists a big performance gap between the performance of the three existing association algorithms and the optimal throughput; these algorithms achieve only $54 \%, 68 \%$, and $81 \%$ throughput compared to the local optimal association control. The results also show that the association control algorithm can be further improved if it has access to future knowledge. Particularly, the offline optimal algorithm with future AP information improves the performance of the local optimal by up to $10 \%$.

The rest of the paper is organized as follows. We present the related work in Section 2, and in Section 3, we describe the system model and problem statement. From the given information of APs, we present the optimal association control problems in Section 4. We evaluate the performance of the online algorithms using simulation in Section 5. We finally conclude our paper in Section 6.

\section{Related work}

Related work falls under two categories: (i) techniques for improving the reliability and performance of vehicular Wi-Fi Internet access, and (ii) studies addressing the association and handoff problems of mobile users.

Several recent studies have explored the Internet access via roadside access points for the moving vehicles. In
[1], Ott and Kutscher identified the characteristics of the drive-thru network access. They show that vehicles experience three different connectivity phases while driving by an access point: the entry phase, production phase, and exit phase. During the entry phase and exit phase, the vehicles suffer from poor connectivity, while in the production phase, it maintains good connection quality. In [2], Hadaller et al. studied the impact of the above three phases on the TCP goodput through detailed experiments in several drive-thru network access scenarios. In [3], Bychkovsky et al. conducted experimental studies with numerous open $\mathrm{Wi}$-Fi access points for vehicular Internet access in urban environments. The experiments observe that the mean connection duration is fairly short (12 to $13 \mathrm{~s}$ ), and it requires high latency to complete the IP address acquisition. This highly affects the application layer performance. Our goal is to improve the application layer performance through the association control techniques.

Recently, there are also efforts to support IP connectivity in vehicular Internet access by utilizing Network Mobility (NEMO) [14-16]. They consider IP-layer handover for vehicular network, but we are dealing with the AP association problem. Ad hoc communication based on relay access has been explored to extend the infrastructure coverage in drive-thru network access [17]. Although we can utilize the ad hoc communication in this paper, for simplicity, we only consider the single-hop communication between vehicles and APs.

There are various approaches to improve the mobile user performance by association control. Association control is a decision process of (i) how to select an AP and (ii) when to conduct handoff to another AP. Typically, mobile users use the channel quality as the association metric for handoff initiation. In [4], Giannoulis et al. proposed a handoff technique that supports vehicular users in the urban mesh network deployed in the southeast part of Houston. They consider AP-quality scores as well as channel quality to associate with the APs. The AP-quality scores are determined by average load and backhaul connectivity. However, they do not consider the connection duration when controlling the handoff frequency. In [11], Kim et al. proposed an association control solution that minimizes the frequency of handoffs. The mobile devices always select the APs with the longest connection time due to the severe handoff overhead, such as association and DHCP latency. In [9], Deshpande et al. developed new handoff and data transfer strategies for moving vehicles in urban areas. They reduce the connection setup latency by using RF fingerprints of APs. The vehicles know the useful APs in the current location beforehand through the RF fingerprint, and thus, the vehicle conducts scripted handoff. In [12], Lei et al. designed an 
efficient association algorithm to achieve total throughput maximization and fairness of all users. Similar to our method, they also presented an optimization problem of association control for efficiency and fairness. However, they do not consider the handoff overhead. In this paper, we deal with the association scheduling problem considering handoff overhead from the vehicle's point of view.

\section{System model}

This section describes the network model and assumptions and then summarizes the notations which are used throughout the paper.

\subsection{System model and assumptions}

We consider a vehicle-to-infrastructure communication system in which vehicles move along roads and opportunistically connect to the roadside Wi-Fi APs for wireless Internet access. Each vehicle adopts an association control mechanism to associate with the APs based on the predefined association criterion (e.g., the AP with the strongest signal strength or the least offered load). Due to the mobility of the vehicle and the limited coverage of APs, the number of available APs to a vehicle is finite and varies over time. The vehicle can associate with only one AP at a given time. A handoff is conducted if it is better to use a new AP compared to the current AP. This handoff may result in throughput gain but incur overhead in terms of delays $^{\mathrm{a}}$.

We assume that vehicles have Global Positioning System (GPS)-equipped devices, so that they know their location, speed, and direction information. When the vehicles get their mobility information and the traffic information of roads on runtime, they can predict their location after some period of time by dead reckoning [18]. We also assume that we have the location information of the roadside APs. In fact, several open or commercial service providers have provided a list of APs and their attributes such as the geographical coordinates, channel numbers, and estimated backhaul capacity $[19,20]$. From the vehicle's mobility prediction and roadside AP's information, they can compute the estimated handoff overhead and effective bit rate. We study an optimal association scheduling method that determines the optimal AP selection sequence by considering the trade-off between the throughput gain from conducting handoff and the resulting overhead.

Without loss of generality, we consider the association control of a target vehicle over the time interval $[0, T]$. Time is divided into discrete time slots that have variable durations $\left[t_{i}, t_{i+1}\right]$, represented by a set of slot index $I=$ $1,2, \ldots, m$, where $0 \leq t_{i}<t_{i+1}<\ldots<t_{m} \leq T$ for time index $i \in I$. At each time slot $i$, the vehicle is given a set of available roadside APs, $J_{i}$, which will be fixed during the given time interval.

Let $r_{i j}$ be the effective bit rate (bit/s) of the link between the vehicle and AP $j$ at time slot $i$. The bit rate $r_{i j}$ may vary over time as the vehicle moves. For simplicity of analysis without losing the key insights of the association scheduling problem, however, we assume that at a given time slot $i$, the effective bit rate $r_{i j}$ is fixed. We also assume that the scheduling algorithms know the effective bit rate $r_{i j}$ at that time. Nevertheless, note that our analytical framework can capture the change of the bit rate by dividing a time slot into several non-overlapping subintervals with different bit rates, at the cost of analytical complexity.

If the AP associated with the vehicle at slot $i+1$ is different from the AP of the duration of slot $i$, then the vehicle is said to have undergone a handoff at slot $i+1$. When the handoff takes place, the mobile users in the vehicle cannot access the Internet for some time duration. We assume that the handoff overhead is constant and $c$ (second) denotes the overhead.

\subsection{Notations}

Prior to the problem formulation, we summarize the indices and notations which are used throughout the paper in the following:

- $i \in I$ index interval, where $I=\{1,2, \ldots, m\}$

- $j \in J$ index APs, where $J=\{1,2, \ldots, n\}$ the set of all the APs

- $J_{i} \subseteq J$ represents available APs in interval $i$

- $s_{i}$ is the length of interval $i$, i.e., $s_{i}=t_{i+1}-t_{i}$

- $r_{i j}$ is the effective bit rate of AP $j$ in interval $i$

- $c$ is the handoff overhead (second)

For each interval $i$, we represent the association decision by the decision variable $x_{i j}$ which denotes whether the target vehicle associates with $\mathrm{AP} j$ at time slot $i$, i.e., $x_{i j}$ (for $i=1,2, \ldots, m$ and $j \in J_{i}$ ) is 1 if the mobile user in the vehicle associates with $\mathrm{AP} j$ in interval $i$, and 0 otherwise.

An example scenario with four APs is illustrated in Figure 1.

For instance, the available APs at time slot 3 are AP1, $\mathrm{AP} 2$, and AP3; thus, $J_{3}=\{\mathrm{AP} 1, \mathrm{AP} 2, \mathrm{AP} 3\}$.

\section{The offline optimal scheduling problem}

Based on the above network model and assumptions, our objective is to derive an optimal association scheduling method that maximizes the throughput of a target vehicle. In this section, we show that computing the offline optimal schedule can be casted into a linear 


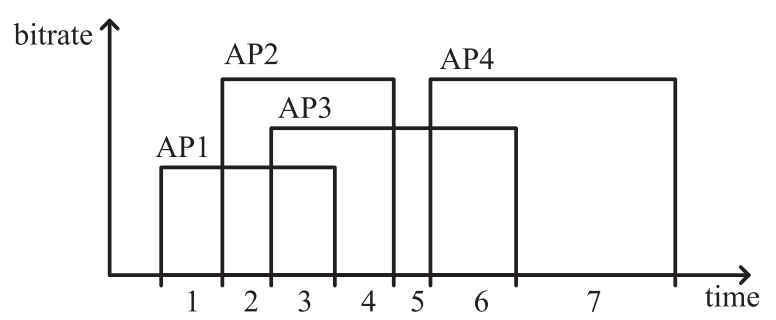

Figure 1 Available APs and handoff decision time.

programming problem, and thus, we have the optimal solution in polynomial time. In addition to the optimal solution that maximizes the throughput, our framework can be extended to obtain the optimality of association that minimizes the frequency of handoffs occurring in vehicles (see Appendix).

\subsection{Objective function}

The objective of the association scheduling is to maximize the total transmitted bits during the time intervals $I=\{1,2, \ldots, m\}$ for the given available APs and effective bit rate information. Computing the optimal association scheduling is equivalent to finding the optimal values for $x_{i j}, i \in I$ and $j \in J_{i}$ by optimizing the trade-off between the throughput gain from a handoff and the resulting re-association overhead $c$.

Therefore, we formulate the offline optimal association control scheduling as a combinatorial optimization problem that finds the optimal values for $x_{i j}\left(i \in I\right.$ and $\left.j \in J_{i}\right)$ with the following objective function:

$$
\begin{aligned}
\max & \sum_{i=1}^{m}\left(s_{i} \cdot\left(\sum_{j \in J_{i}} r_{i j} x_{i j}\right)\right. \\
& \left.-c \cdot\left(\sum_{j \in J_{i} \cap \cap_{i-1}} r_{i j} x_{i j} \cdot\left(x_{i j}-x_{i-1 j}\right)+\sum_{j \in J_{i}-J_{i-1}} r_{i j} x_{i j}\right)\right)
\end{aligned}
$$

subject to

$$
\begin{gathered}
\sum_{j \in J_{i}} x_{i j} \leq 1, \quad \forall i \in I, \\
x_{i j} \in\{0,1\} .
\end{gathered}
$$

The objective function given in Equation 1 consists of two terms. The first term denotes the total amount of transmitted bits. The re-association overhead is reflected by the second term. Note that the re-association overhead should be considered only when a handoff occurs. In other words, if the AP $j$ used in interval $i-1$ is continuously used in interval $i$ (i.e., $x_{i-1 j}=x_{i j}$ ), then there is no reassociation overhead. On the contrary, if the mobile user selects a new AP $j^{\prime}$ which was not used in interval $i-1$ (i.e., $x_{i-1 j}=1, x_{i j}=0$, yielding $x_{i-1 j}-x_{i j}=1$ ), the vehicle cannot transmit data during $c$ seconds due to the handoff overhead. Thus, we express the re-association overhead as

$$
c \cdot\left(\sum_{j \in J_{i} \cap J_{i-1}} r_{i j} \cdot x_{i j} \cdot\left(x_{i j}-x_{i-1 j}\right)+\sum_{j \in J_{i}-J_{i-1}} r_{i j} \cdot x_{i j}\right) .
$$

Also, Equation 2 is provided since the vehicle can associate with at most one AP among the available APs according to the system model given above.

\subsection{Computing the optimal scheduling problem}

The objective function given in Equation 1 is a non-linear integer programming problem with binary integer decision variable $x_{i j}$. Although the optimal solution of the problem is a subset of all combinatorial and thus is solvable by brute-force enumerative algorithms [21], its calculation complexity is too high to be practical.

To address this challenging issue, we transform the above non-linear function into a linear function by exploiting the properties of the binary decision variable.

Since the decision variable $x_{i j}$ is a binary value from which it is given $x_{i j}^{2}=x_{i j}$, we rewrite the non-linear component in the objective function of Equation 1 as

$$
x_{i j} \cdot\left(x_{i j}-x_{i-1 j}\right) \Rightarrow x_{i j}^{2}-x_{i j} \cdot x_{i-1 j} \Rightarrow x_{i j}-x_{i j} \cdot x_{i-1 j} .
$$

Then, by setting $z_{i j}=x_{i j} \cdot x_{i-1 j}$, we view $z_{i j}$ as another binary variable. This yields two additional constraints in the objective function that reflect the same handoff costs:

$$
\begin{cases}z_{i j} \leq x_{i j}, & \forall i \in I, j \in J_{i} \cap J_{i-1}, \\ z_{i j} \leq x_{i-1 j}, & \forall i \in I, j \in J_{i} \cap J_{i-1} .\end{cases}
$$

Thus, the optimization problem is rewritten as

$$
\begin{aligned}
\max & \sum_{i=1}^{m}\left(s_{i} \cdot\left(\sum_{j \in J_{i}} r_{i j} x_{i j}\right)\right. \\
& \left.-c \cdot\left(\sum_{j \in J_{i} \cap J_{i-1}} r_{i j}\left(x_{i j}-z_{i j}\right)+\sum_{j \in J_{i}-J_{i-1}} r_{i j} x_{i j}\right)\right)
\end{aligned}
$$


subject to

$$
\begin{aligned}
& \sum_{j \in J_{i}} x_{i j} \leq 1, \quad \forall i \in I, \\
& z_{i j} \leq x_{i j}, \quad \forall i \in I, j \in J_{i} \cap J_{i-1}, \\
& z_{i j} \leq x_{i-1 j}, \quad \forall i \in I, j \in J_{i} \cap J_{i-1}, \\
& x_{i j} \text { and } z_{i j} \in\{0,1\} .
\end{aligned}
$$

However, it is still non-trivial to solve the above integer program (IP) because of the integrality constraints. We will discuss the property of the objective function and show that its solution can be obtained in polynomial time.

Let us consider a IP $\max \left\{c^{T} x: A x \leq b, x \in \mathbb{Z}_{n}\right\}$ and its linear programming (LP) relaxation $\max \left\{c^{T} x: A x \leq\right.$ $\left.b, x \in \mathbb{R}_{n}\right\}$. A linear program in real variables is said to be integral if it has at least one optimal solution which is integral. Likewise, a polyhedron $\mathbf{P}=\{x: A x \leq b\}$ is said to be integral if the linear program has an optimum solution $x^{*}$ with integer coordinates. According to the LP theory [22], solving an IP over an integral polyhedron can be done in polynomial time through LP relaxation of integral constraints, and then the LP has an integral optimal solution.

One common way of proving that a polyhedron is integral is to show that its constraint matrix is TU [21], where a matrix is said to be totally unimodular if the determinant of each square submatrix is $0,+1$, or -1 . This leads to the following proposition.

Proposition 1. If a matrix $A$ is totally unimodular and $b$ is an integral vector, then the polyhedron $P=\{x: A x \leq b\}$ is integral.

\section{Proof. See [21].}

We now show that the constraint matrix of the objective function given in Equation 7 satisfies TU. At this point, however, it is not easy to recognize that the constraint matrix in Equation 7 is TU by directly using the definition of TU. Thus, we consider a general sufficient condition with the following theorem.

\section{Theorem 1. A matrix $A$ is totally unimodular if}

(i) $a_{p q} \in\{0,+1,-1\}$ for all $p, q$, and

(ii) for any subset $M$ of the rows of $A$, there exists a partition $\left(M_{1}, M_{2}\right)$ of $M$ such that each column $q$ satisfies

$$
\left|\sum_{p \in M_{1}} a_{p q}-\sum_{p \in M_{2}} a_{p q}\right| \leq 1 .
$$

Proof. See [22].
With a more general condition in Theorem 1, we will show that the constraint matrix of the linear integer problem given in Equation 7 is totally unimodular. We first rewrite the constraints of the linear integer problem given in Equation 8 in the form of the standard formation as

$$
\begin{aligned}
\sum_{j \in J_{i}} x_{i j} & \leq 1, \\
-x_{i j}+z_{i j} & \leq 0, \\
-x_{i-1 j}+z_{i j} & \leq 0 .
\end{aligned}
$$

Then, one can easily express the above constraint as the form of $A \mathbf{x} \leq b$, where $A$ denotes the constraint matric, defined as $A \triangleq\left[a_{p q}\right], \mathbf{x} \triangleq(x, z)^{T}$ and $b \triangleq\left(b_{q}\right)$ such that $b_{q} \geq 0$. Then, we have the following theorem.

Theorem 2. Let $A$ be the constraint matrix of the linear integer problem given in Equation 7 (i.e., in the form of $A x \leq b)$, then matrix $A$ is totally unimodular.

Proof. We first introduce a partitioning method that obtains a partition $\left(M_{1}, M_{2}\right)$ for an arbitrary subset $M$ of the rows of $A$. Let $B_{i}$ be a subset of $A$ such that $B_{i}$ includes all the rows corresponding to the coefficients of $x_{i j}$ in the constraints given in Equation 8. Let $Q_{i}^{1}, Q_{i}^{2}$, and $Q_{i}^{3}$ denote the submatrices of $A$ containing all the rows corresponding to each constraint in Equations 8a, $8 \mathrm{~b}$, and $8 \mathrm{c}$ for interval $i$, respectively. Then, $B_{i}$ is given as $B_{i}=Q_{i}^{1} \cup Q_{i}^{2} \cup Q_{i+1}^{3}$. For an arbitrary subset $M$, Algorithm 1 generates a partition $\left(M_{1}, M_{2}\right)$ by iteratively testing every row in $B_{i} \cap M$ and assigning the row into $M_{1}$ or $M_{2}$ for $i \in I$. It is straightforward to show that this portioning rule obtains a partition $\left(M_{1}, M_{2}\right)$ of $M$ correctly.

The basic principle of how to assign each testing row is as follows. For two different rows $p$ and $p^{\prime}$ which are both correspondent to interval $i$, we perform the partition according to two elements $a_{p q}$ and $a_{p^{\prime} q}$ in the same column $q$. For the case that both $a_{p q}$ and $a_{p^{\prime} q}$ are 1 (or -1 ), we assign such rows $p$, and $p^{\prime}$ to different partitions $M_{1}$ and $M_{2}$, respectively. If both $a_{p q}$ and $a_{p^{\prime} q}$ are non-zero but their signs are different, i.e., $\left(a_{p q}, a_{p^{\prime} q}\right)=\{1,-1\}$ or $\{-1,1\}$, then we assign such rows to the same partition $M_{1}$ or $M_{2}$. Since $B_{i} \cap M$ and $B_{i-1} \cap M$ can have nonzero values in the same column due to the third constraint (8.c), we consider the rows in $M_{1}$ and $M_{2}$ already assigned for interval $i-1$ when assigning the rows of $B_{i} \cap M$ for interval $i$. Thus, we assign the rows of $B_{i} \cap M$ into $M_{1}$ or $M_{2}$ depending on whether any non-zero value in the same column was already assigned in both $M_{1}$ (or $M_{2}$ ) and $B_{i} \cap M$ or not. The partitioning rule is described in Algorithm 1. 


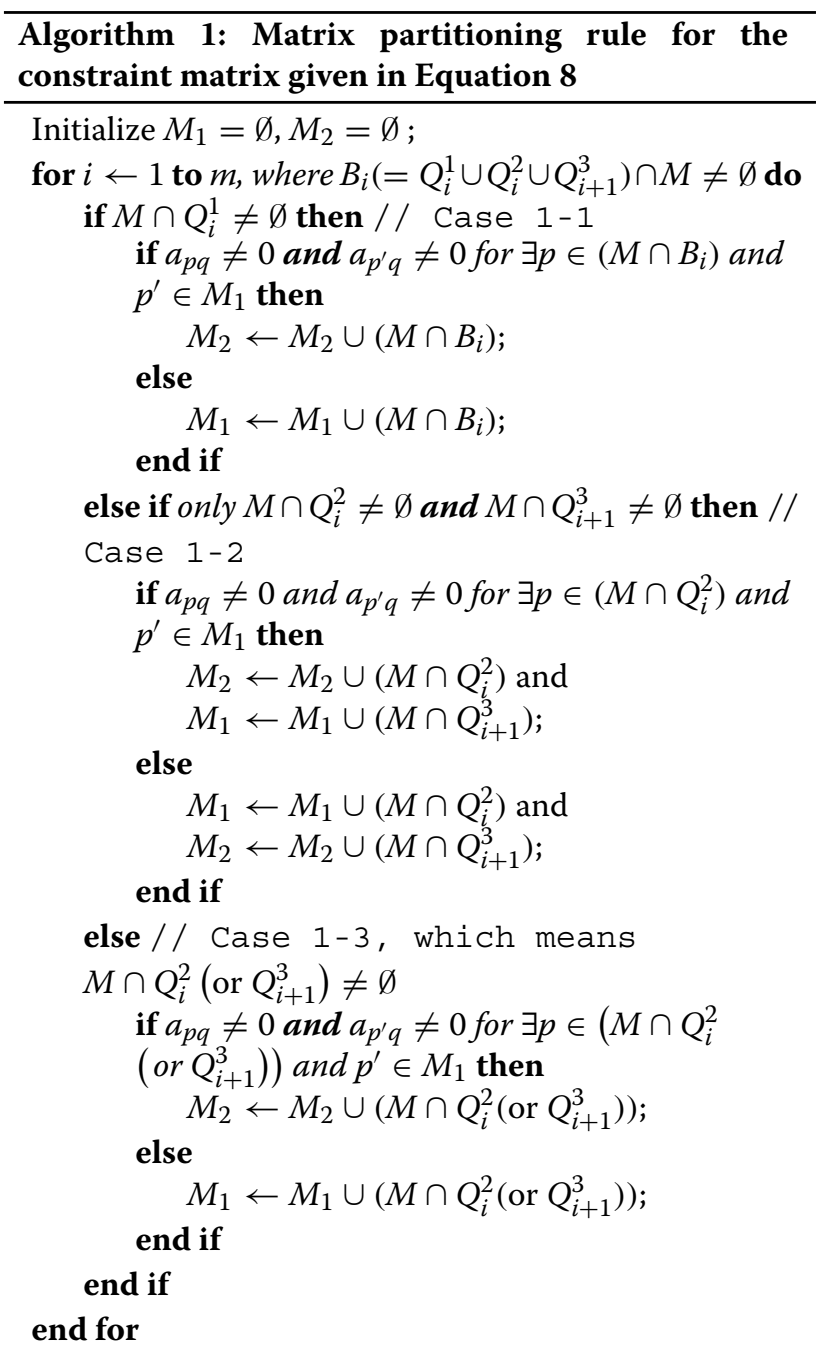

For a given arbitrary subset $M$ of rows of $A=\left[a_{p q}\right]$, we can have its partition $\left(M_{1}, M_{2}\right)$ based on Algorithm 1 . Since condition (i) of Theorem 1 is already satisfied in Equation 8, we only need to show that the partition $\left(M_{1}, M_{2}\right)$ will satisfy condition (ii) of Theorem 1 .

We can easily see that the number of non-zero entries $a_{p q}$ on column $q$ for all $p \in M$ is at most three from Equation 8. Therefore, the number of non-zero entries on the same column in $M_{1}$ and $M_{2}$ is at most three. Let $N_{q}$ denote the number of non-zero entries either in $M_{1}$ or in $M_{2}$ for column $q$.

(i) $N_{q}=0,1$ : nothing to prove.

(ii) $N_{q}=2$ : There are three subcases for $\left(a_{p q}, a_{p^{\prime} q}\right)$ where $p \neq p^{\prime}$ :

(ii-1) $\left(a_{p q}, a_{p^{\prime} q}\right)=\{-1,-1\}$ : In this case, $p \in Q_{i}^{2}$ and $p^{\prime} \in Q_{i+1}^{3}$ (or $p^{\prime} \in Q_{i}^{2}$ and $p \in Q_{i+1}^{3}$ ) for $\exists i$. This happens only if $B_{i} \cap M \neq \emptyset$ by the definition of $B_{i}$. Thus, it is belonging to the case 1-2 in Algorithm 1; the two rows $p$ and $p^{\prime}$ were partitioned to different partitions. Thus, $\left|\sum_{p \in M_{1}} a_{p q}-\sum_{p \in M_{2}} a_{p q}\right| \leq 1$ holds.

(ii-2) $\{1,-1\}$ (or $\{-1,1\}$ ): Since $p \in Q_{i}^{1}$ for $\exists i$, and this is belonging to case 1-1 in Algorithm 1, the two rows $p$ and $p^{\prime}$ in $B_{i} \cap M$ should be assigned to the same partition $M_{1}$ or $M_{2}$. Thus, $\left|\sum_{p \in M_{1}} a_{p q}-\sum_{p \in M_{2}} a_{p q}\right| \leq 1$ holds.

(iii-3) $\{1,1\}$ : This can only happen for a column with respect to $z_{i j}$ for $\exists i\left(B_{i-1}\right.$ and $\left.B_{i}\right)$. Then, the columns of $M_{1}$ (or $M_{2}$ ) and $B_{i} \cap M$ are belonging to the case 1-2 in Algorithm 1; thus, the two rows $p$ and $p^{\prime}$ are assigned to different partitions. Thus,

$\left|\sum_{p \in M_{1}} a_{p q}-\sum_{p \in M_{2}} a_{p q}\right| \leq 1$ holds.

(iii) $N_{q}=3$ : In this case, among three non-zero $a_{p q}$, two of them should be -1 and the other one is +1 where $p \in M$. The partition $\left(M_{1}, M_{2}\right)$ is performed according to case 1-1 in Algorithm 1, and the summation of $1,-1,-1$ is -1 . Thus, $\left|\sum_{p \in M_{1}} a_{p q}-\sum_{p \in M_{2}} a_{p q}\right| \leq 1$ holds.

In all cases, non-zeros in a column for the partition $\left(M_{1}, M_{2}\right)$ obtained from Algorithm 1 satisfy condition (ii) of Theorem 1. Therefore, the constraint matrix $A$ is totally unimodular.

Solving an IP over an integral polyhedron $\mathbf{P}$ can be done in polynomial time. Therefore, from the results of Theorems 1 and 2, we can obtain the solution of the integer program given in Equation 8 in polynomial time.

Remark. In addition to the optimal scheduling problem that maximizes throughput, our framework can be extended to obtain the optimality of association that minimizes the frequency of handoffs occurring in vehicles. In the Appendix, we formulate the optimal handoff minimization scheduling problem as a non-linear integer programming and show that this problem also holds the TU property, and thus is solvable in polynomial time.

\section{Performance comparison of online algorithms with perfect link information}

The optimal association scheduling solution renders practical challenges since it requires perfect future knowledge, such as the set of available roadside APs, the available link duration (connection time), and the effective bit rate between the vehicle and the roadside APs. Nevertheless, the optimal scheduling solution still provides an important insight. Further, it can also be used as a baseline to compare the performance of various online association control algorithms. 
In this section, we evaluate the performance of three existing online algorithms by comparing them with the optimal association algorithm given in Section 4 in a mobile wireless network via real bus trace-driven simulations. We first describe the evaluation methodology for the performance study, then discuss the evaluation results that compare the performance of the optimal association with conventional association algorithms using various handoff metrics.

\subsection{Methodology}

\subsubsection{Association control algorithms under consideration}

We compare three association control schemes with the optimal solution: (i) bandwidth-based association, (ii) duration-based association, and (iii) combined metricbased association. We give some explanations for these schemes as follows:

- Bandwidth-based association (Ba): The most widely used association control method is to use some measurable metrics (e.g., signal strength or collision probability) that infer the achievable throughput. We study the online association control algorithm proposed in [23], in which the mobile nodes select the AP with the largest effective bit rate whenever it meets a new AP or the current associated AP is disconnected. This greedy decision-based algorithm does not consider the handoff overhead, thus suffering from frequent handoffs.

- Duration-based association (Du): There have been some research efforts to alleviate the handoff latency problem. We study the online greedy algorithm proposed in [11] in which the mobile nodes select the AP with the longest duration whenever the current AP becomes unavailable. In [11], this greedy online algorithm is optimal with an offline setting where the perfect future knowledge is given.

- Combined metric-based association ( $\mathrm{BaDu})$ : The association control scheme proposed in [24] exploits the trade-off between the bandwidth-based association and duration-based association algorithms. The mobile node selects the AP with the highest bandwidth duration whenever the mobile node meets new APs or the current associated AP becomes unavailable.

Note that the performance of these online association control algorithms significantly hinges on how they can accurately estimate the required metrics, such as available bandwidth and connection duration. However, our focus in this section is not to study the accuracy of the link information measurement, but to compare various online scheduling algorithms with the optimal association control algorithm and extract the relative merits of these algorithms. Therefore, we assume that these online algorithms have perfect knowledge of the link between the vehicle and available APs, e.g., the effective bit rate and connection time information ${ }^{\mathrm{b}}$, so that we focus on characterizing their difference induced from their different handoff criteria.

\subsubsection{Online optimum and offline optimum}

We use the solution presented in Section 4 as the optimal association control algorithm. We categorize the solution into two algorithms - (i) offline optimal and (ii) local optimal - depending on the availability of future knowledge.

- Local online optimal association (LO): The local optimal ( $L O)$ is an online algorithm where only $J_{i}$ is given at slot $i$ without any knowledge of future sets $J_{i+1}, J_{i+2}, \cdots, J_{m}$. Therefore, with the local online optimal algorithm, a mobile user schedules the optimal association sequence only with a set of current available APs according to the presented method in Section 4. The mobile user reschedules the association sequence whenever the mobile node meets new APs or the current associated AP becomes unavailable.

- Offline optimal association (Optimal): The offline optimal algorithm is given $J_{i}$ information for $\forall i \in I$, i.e., all sets of available $J_{i}$ are given in advance.

\subsubsection{Simulation setup}

We generate realistic mobility scenarios by employing the measurements of real bus traces. We gathered the trace information for over a month period by using the Seoul TOPIS system [13] in which the real-time locations of Seoul Metro buses are publicly available. We compiled the trajectory information of about 7,000 buses. We refine the coordinates of the buses so that the coordinates are mapped into the $10 \mathrm{~km} \times 10 \mathrm{~km}$ road map shown in Figure 2. The update period of each bus' location is $1 \mathrm{~min}$, so we complement the hidden intermediate trajectories of buses based on their average velocities. In Figure 2, the black lines indicate the bus routes, and red points represent the commercial Wi-Fi AP locations. We obtained the locations of roadside APs from an open Wi-Fi database named Olleh WiFi zone [25]. We sampled hundreds of AP locations around the simulation area.

\subsection{Comparison results and discussion}

To solve the optimization problem, we use the General Algebraic Modeling System (GAMS) [26], a numerical tool specially designed for modeling large and complex optimization problems. First, we measure the execution time required to solve the optimal association problem using GAMS. We use a desktop equipped with Intel(R) Core 2 Quad CPU 2.4 GHz and 4G RAM. Table 1 shows the 

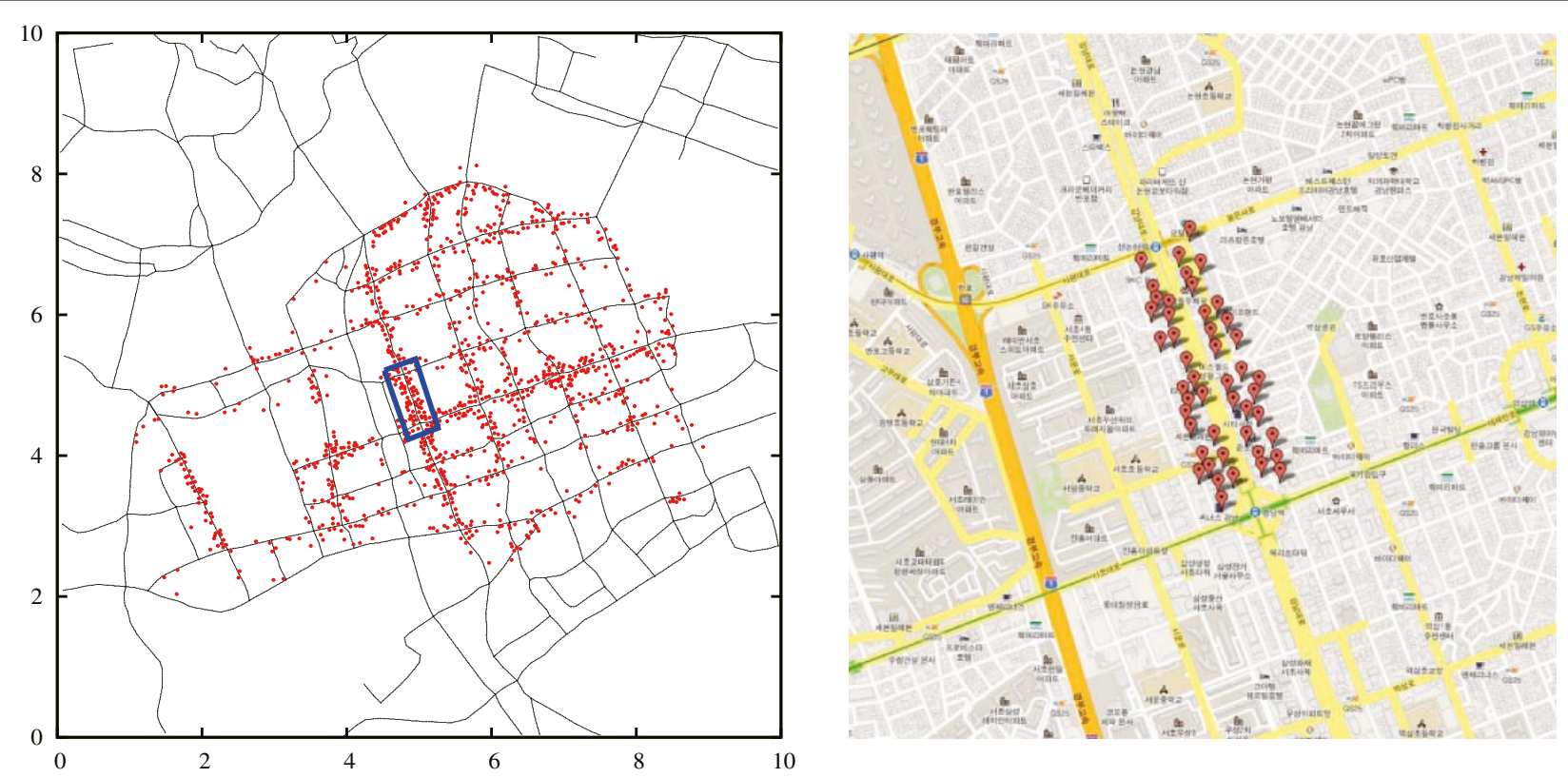

Figure $210 \mathrm{~km} \times 10 \mathrm{~km}$ area in Seoul.

execution time for the optimal association for various problem sizes. We observe that despite the large number of variables, the computations for the integer problems are completed no later than $1 \mathrm{~s}$. This is because the TU condition of the optimal association problem provides LP relaxation which is equivalent to the original IP problem.

To evaluate the throughput maximization problem, we selected a road segment as shown in Figure 2 and utilized the 1-h movement traces of 434 buses passing this area. Then, based on the association metric of each association control algorithm, we scheduled the association of 434 buses. We set the effective bit rate $r$ according to the geographical distance between the buses and the Wi-Fi APs, i.e., for the short link distance, we use high bit rate and vice versa. In the network model explained in Section 3, the bit rate is known to each bus and fixed for a given time slot. The re-association overhead is set to $2 \mathrm{~s}(c=2)$.

Figure 3 shows the normalized transmitted bits of five association control algorithms: bandwidth-based association $(B a)$, duration-based association $(D u)$, combined metric-based association $(B a D u)$, local optimum association (LO), and offline optimal association (Optimal) algorithms. We observe that the duration-based association $(D u)$ shows the worst performance compared to

Table 1 Execution time for solving optimal association

\begin{tabular}{ccc}
\hline Number of APs & Time (s) & Number of variables \\
\hline 50 & 0.016 & $98 \times 50 \times 2$ \\
100 & 0.063 & $193 \times 100 \times 2$ \\
200 & 0.296 & $574 \times 300 \times 2$ \\
300 & 0.843 & $976 \times 500 \times 2$ \\
\hline
\end{tabular}

the other online algorithms. The main reason is that the duration-based algorithm connects to the APs with longer available link durations for less frequent re-association, without considering the effective bit rate. As a result, the duration-based algorithm suffers from low throughput once the users associate with an AP with low bandwidth. The bandwidth-based association is shown to achieve a relatively high bit rate compared to the duration-based association. However, we clearly observe that it experiences frequent handoffs, because the connection duration is not considered, and thus, it cannot achieve the best performance. The combined metric-based association control algorithm $(B a D u)$, is shown to achieve the highest performance among the three online association control algorithms. This is mainly because it considers the link bandwidth as well as the available link duration and

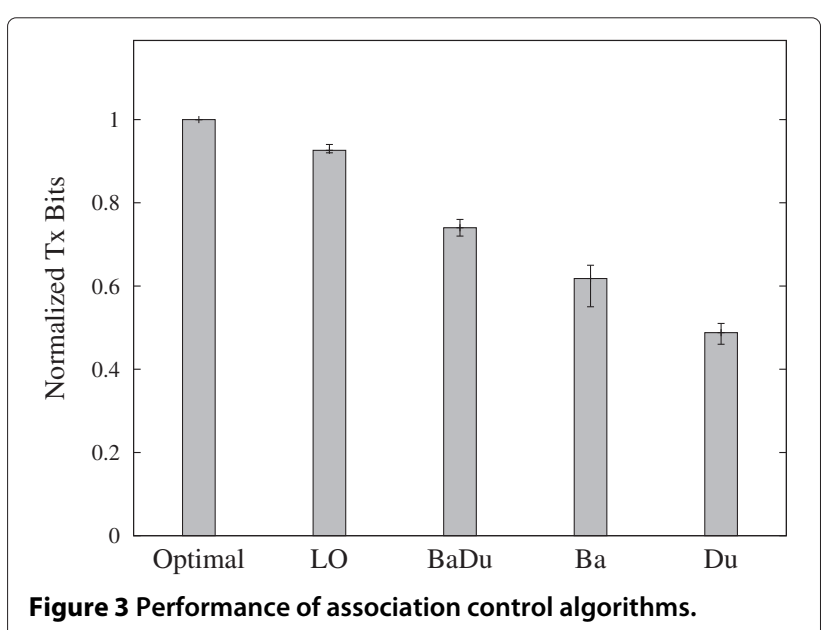


exploits their trade-off. However, we observe that it sometimes selects the AP that has low bit rates but with a very long connection duration, and suffers from low throughput. From Figure 4, we can see that these three algorithms achieve reasonable performance, but there is a large performance gap between them and the optimal association control. The bandwidth-based association $(B a)$, duration-based association $(D u)$, and combined metricbased association $(\mathrm{BaDu})$ achieve only $54 \%, 68 \%$, and $81 \%$ throughput compared to the local optimal association control $(L O)$. We also observe that the association control algorithm can be further improved if it has access to future knowledge. Particularly, the offline optimal (Optimal) improves the performance of local optimal $(L O)$ by up to $10 \%$.

Next, we study the impact of the degree of future information on the performance. We compare the optimal solutions with different degrees of future information to the offline optimal algorithm. In Figure 4, we compare three optimal solutions having 5, 10, and $15 \mathrm{~s}$ of future knowledge, denoted by $\mathrm{LOe}(5), \mathrm{LOe}(10)$, and $\mathrm{LOe}(15)$, respectively. For example, in the simulation, buses with $\mathrm{LOe}(5)$ algorithm are given $5 \mathrm{~s}$ of future AP information, and they schedule the association according to the optimal solution presented in Section 4 using the future information together as its input parameters. One interesting observation is that if we predict the AP information $15 \mathrm{~s}$ ahead in the future, we achieve $97 \%$ compared to the offline optimal performance.

Next, we evaluate the effect of handoff overhead. Figure 5 shows the normalized transmitted bits of the online algorithms as a function of various handoff overhead. With lower handoff overhead, the performance of the online algorithms becomes close to that of the optimum algorithm, whereas with higher handoff overhead, there is a large performance gap. If the handoff overhead is low, it is likely beneficial to switch to the AP with higher

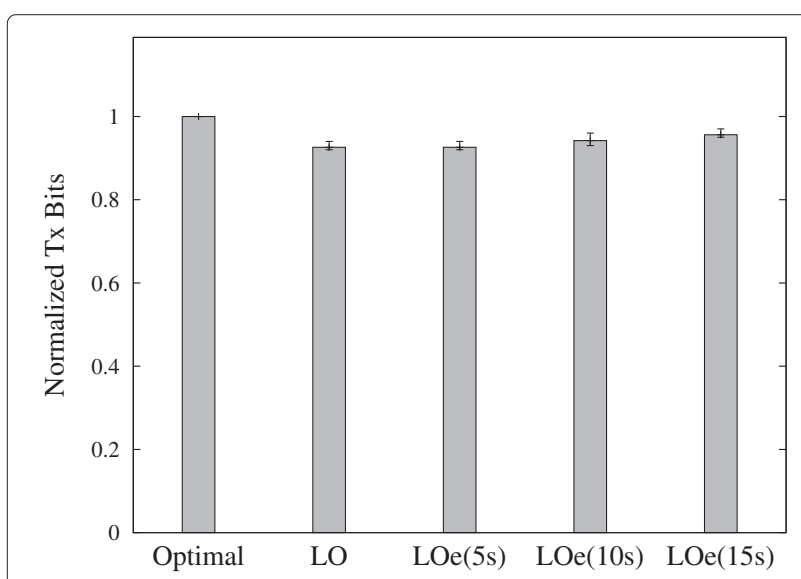

Figure 4 Performance of optimal solutions with variable future knowledge. bit rate whenever vehicles meet new APs. This straightforward intuition can be explained naturally by the result shown in Figure 5. We further observe that the available bandwidth of APs becomes the critical factor for the association decision with lower handoff overhead, whereas the link duration with APs becomes more important for higher handoff overhead. Thus, $B a$ achieves high throughput with lower handoff overhead, while $D u$ performs better with higher handoff overhead.

In addition to the throughput maximization problem, we evaluate the handoff minimization problem, where its detailed solution is given in the Appendix. Figure 6 shows the normalized number of handoffs of the algorithms. For this study, we consider an algorithm, $B a$ (until), extending the bandwidth-based algorithm $B a$. It associates with APs having the highest effective bit rate and keeps the association until the AP becomes unavailable. As shown in the figure, association by not considering connection duration causes frequent handoffs. From the result shown in Figure 6, we see that an online algorithm $\mathrm{Du}$ presented in [11] achieves the optimal throughput equal to the optimal solution in terms of the number of handoffs. This is because $D u$ selects new APs only when the current association is broken, and then it chooses the AP with the longest duration. Therefore, if we obtain the duration information between APs and mobile nodes, we can perform the optimal scheduling with low computation complexity.

\section{Conclusions}

In this paper, we studied the optimal association control techniques for the vehicular Wi-Fi networks. The tradeoff between using the effective bit rate and available link duration of APs as an association metric motivates the need for an efficient association control scheme. We have introduced a theoretical framework for optimal association control and prove that the optimization problem is solved in polynomial time. We performed extensive simulation studies to compare the existing online association control schemes with the obtained optimal control. We observed that there exists a large performance gap between the performance of the three existing association algorithms and the optimal throughput. We have shown that the association control algorithm can be further improved if it has access to future knowledge. Particularly, the offline optimal with future AP information improves the performance of local optimal by up to $10 \%$.

\section{Endnotes}

${ }^{a}$ The handoff overhead usually consists of association delay, IP acquisition delay (DHCP delay), and ARP lookup delay [9].

${ }^{b}$ Note that several advanced estimation mechanisms have been proposed [24], so that the above online 


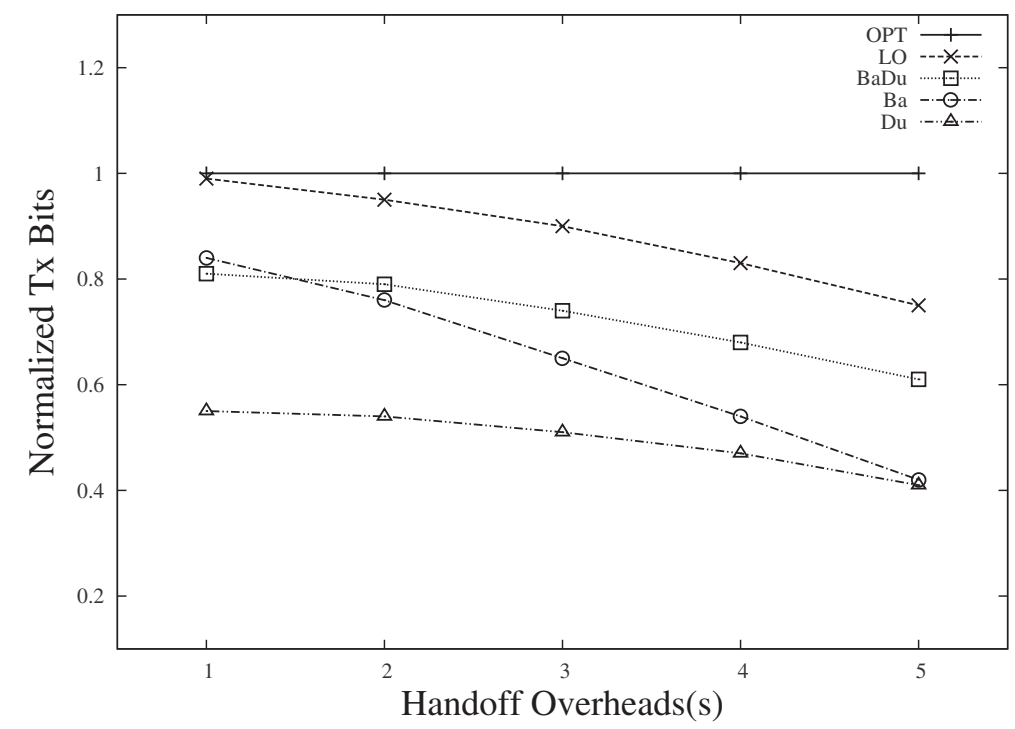

Figure 5 The effect of handoff overheads.

algorithms can acquire the link information in practice. For example, the IEEE 802.11 beacon message of roadside APs can be used to help moving vehicles obtain the link information. The beacon message has reserved fields which may include vendor-specific information. Thus, the APs can deliver information, such as the current location and backhaul capacity, through the reserved fields in the beacon message. The client vehicle that receives the beacon message estimates the duration through the above equations.

\section{Appendix}

\section{Handoff minimization}

In this section, we tackle the problem of minimizing handoffs. As vehicles move along their route, they continuously

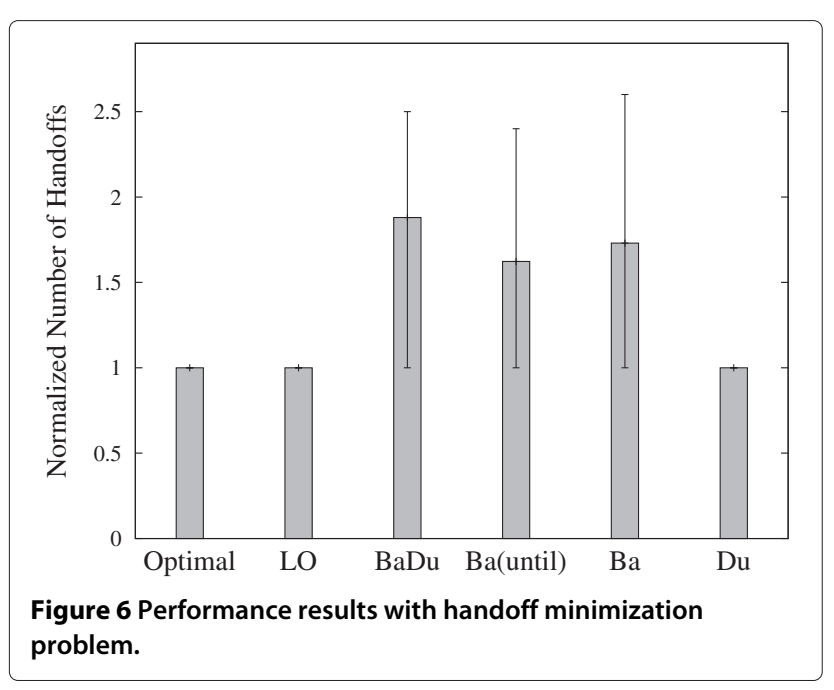

keep associating with different APs to maintain the connectivity to the Internet. However, such frequent handoffs may cause unacceptable service interruption. Therefore, it is desirable to take an approach to derive the optimal association scheduling that minimizes the handoff frequency. The definitions of the notations used in this section can be found in the previous sections.

Problem formulation: For the given available AP information, we formulate the association control scheduling problem as a integer problem with the following objective function:

$$
\min \sum_{i=1}^{m}\left(\sum_{j \in J_{i} \cap J_{i-1}}\left(x_{i j}-z_{i j}\right)+\sum_{j \in J_{i}-J_{i-1}} x_{i j}\right)
$$

subject to

$$
\begin{aligned}
& \sum_{j \in J_{i}} x_{i j}=1, \quad \forall i \in I \\
& z_{i j} \leq x_{i j}, \quad \forall i \in I, j \in J_{i} \cap J_{i-1} \\
& z_{i j} \leq x_{i-1 j}, \quad \forall i \in I, j \in J_{i} \cap J_{i-1} \\
& x_{i j} \text { and } z_{i j} \in\{0,1\}
\end{aligned}
$$

where $z_{i j}=x_{i j} \cdot x_{i-1 j}$ which is equivalent to the settings in the throughput maximization problem given in Equation 7.

Problem computation: The objective here is to show that the constraint matrix of the linear program in Eq. 9 is totally unimodular; thus, we can obtain its optimal solution in polynomial time. Therefore, we rewrite the 
above constraints into standard formation, i.e., $A \mathbf{x} \leq b$, as follows:

$$
\begin{aligned}
\sum_{j \in J_{i}} x_{i j} & \leq 1, \\
\sum_{j \in J_{i}}-x_{i j} & \leq-1, \\
-x_{i j}+z_{i j} & \leq 0, \\
-x_{i-1 j}+z_{i j} & \leq 0 .
\end{aligned}
$$

Let $Q_{i}^{1-1}, Q_{i}^{1-2}, Q_{i j}^{2}$, and $Q_{i j}^{3}$ be the submatrices of $A$ containing all the rows corresponding to each constraint in Equations 10a, 10b, 10c, and 10d for interval $i$, respectively (for brevity, we reuse the notations in the previous theorem but they represent different constraints). Then, we introduce Algorithm 2 which is a partitioning algorithm for the constraints similar to Algorithm 1.

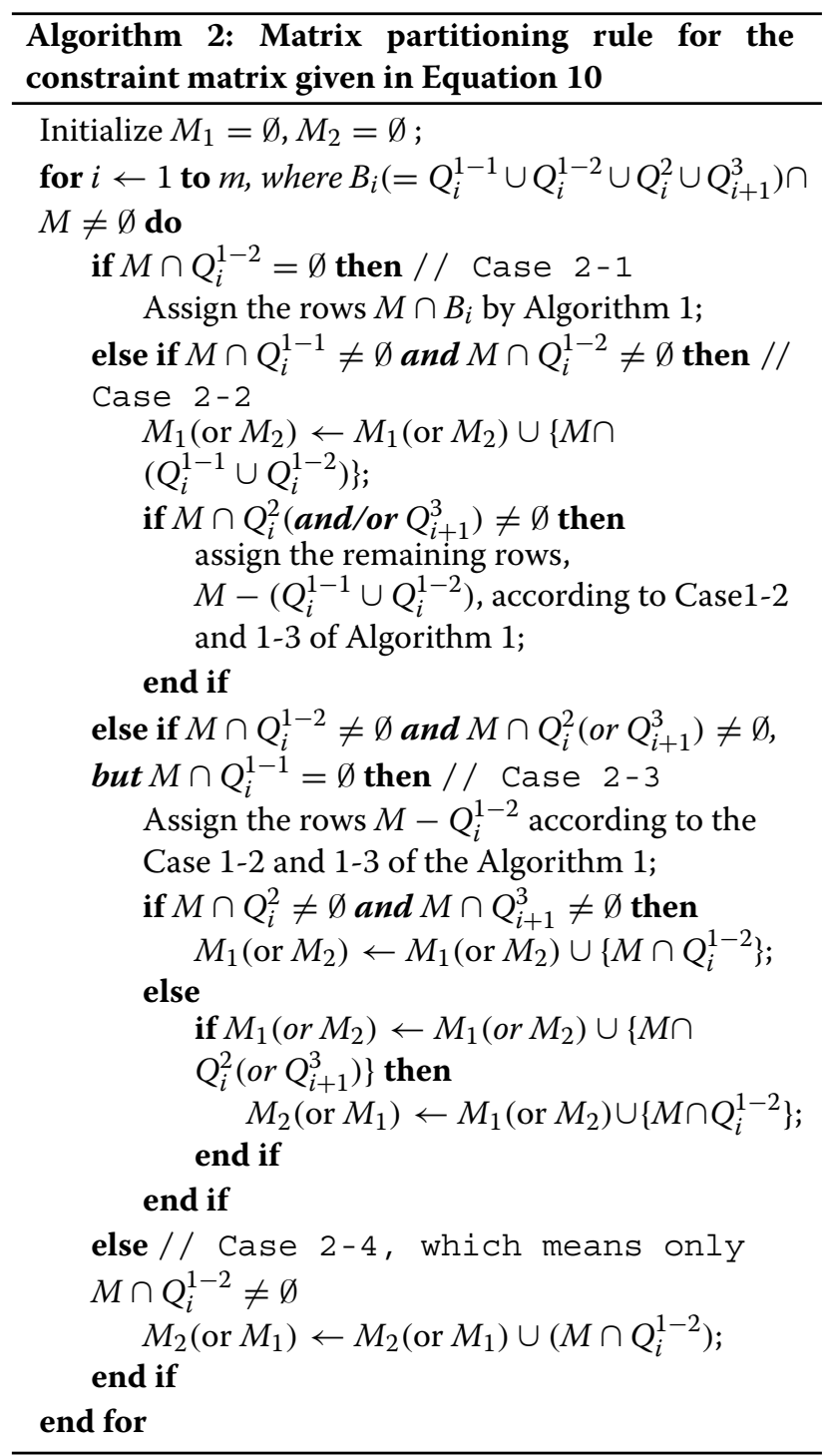

Then, we have the following theorem.

Theorem 3. Let $A$ be the constraint matrix given in Equation 10, then A is totally unimodular.

Proof. For a given arbitrary subset $M$ of rows of $A \equiv$ $\left(a_{p q}\right)$, we have a partition $\left(M_{1}, M_{2}\right)$ of $M$ by using the portioning Algorithm 2. Since condition (i) of Theorem 1 is already satisfied in Equation 10, we only need to show that the partition $\left(M_{1}, M_{2}\right)$ will satisfy condition (ii) of Theorem 1. We will consider the cases for a column $\mathrm{q}$ according to the number of non-zero entries among $a_{p q}$ where $p \in M$, denoted by $N_{q}$. Here, we have at most four non-zero entries for any column $q$, i.e., $N_{q} \leq 4$.

(i) $N_{q}=0,1$ : nothing to prove.

(ii) $N_{q}=2$ : There are three cases for $\left(a_{p q}, a_{p^{\prime} q}\right)$ where $p \neq p^{\prime}$

(ii-1) $\left(a_{p q}, a_{p^{\prime} q}\right)=\{-1,-1\}$ : There are two possible subcases: (1) $p \in Q_{i}^{2}$ and $p^{\prime} \in Q_{i+1}^{3}$ for $\exists i$, (2) $p \in Q_{i}^{1-2}$ and $p^{\prime} \in Q_{i+1}^{3}$ (or $Q_{i}^{2}$ ) for $\exists i$. By the definition of $B_{i}$, this is treated in $M \cap B_{i} \neq \emptyset$, and by case 2-2 and case 2-3 of Algorithm 2, the two rows $p$ and $p^{\prime}$ are partitioned to different partitions. Thus, $\left|\sum_{p \in M_{1}} a_{p q}-\sum_{p \in M_{2}} a_{p q}\right| \leq 1$ holds.

(ii-2) $\{1,-1\}$ : In this case, it is given that $p \in Q_{i}^{1-1}$ and $p^{\prime} \in Q_{i}^{1-2}, Q_{i}^{2}$, or $Q_{i+1}^{3}$. Since $p \in Q_{i}^{1-1}$ for $\exists i$, and this is dealt with case 2-1 and case 2-2, all rows in $M \cap B_{i}$ are assigned to the same partition. Thus,

$\left|\sum_{p \in M_{1}} a_{p q}-\sum_{p \in M_{2}} a_{p q}\right| \leq 1$ holds.

(iii-3) $\{1,1\}$ : This can happen for a column with respect to $z_{i j}$ for $B_{i-1}$ and $B_{i}$. By checking the columns of $M_{1}$ (or $M_{2}$ ) and $M \cap B_{i}$ in each case of Algorithm 1, they are assigned to a different partition $M_{1}$ or $M_{2}$. Thus, $\left|\sum_{p \in M_{1}} a_{p q}-\sum_{p \in M_{2}} a_{p q}\right| \leq 1$ holds.

(iii) $N_{q}=3$ : In this case, we have two possible cases of $a_{p q}, a_{p^{\prime} q}, a_{p^{\prime \prime} q}$ for three rows $p, p^{\prime}$, and $p^{\prime \prime}$.

(iii-1) $\{1,-1,-1\}$ : There are two subcases: (1) $p \in Q_{i}^{1-1}, p^{\prime} \in Q_{i}^{1-2}$ and $p^{\prime \prime} \in Q_{i+1}^{3}\left(\right.$ or $\left.Q_{i}^{2}\right)$ or (2) $p \in Q_{i}^{1-1}, p^{\prime} \in Q_{i}^{2}$ and $p^{\prime \prime} \in Q_{i+1}^{3}$. In the case of (1), case 2-2 of Algorithm 2 assigns rows $\left\{p, p^{\prime}, p^{\prime \prime}\right\}$ into $\left\{p, p^{\prime}\right\} \in M_{1}$ (or $\left.M_{2}\right)$ and $\left\{p^{\prime \prime}\right\} \in M_{1}\left(\right.$ or $\left.M_{2}\right)$ or $\left\{p, p^{\prime}, p^{\prime \prime}\right\} \in M_{1}\left(\right.$ or $\left.M_{2}\right)$. On the other hand, in case of the (2), all rows are assigned to the same partition $M_{1}$ or $M_{2}$ by case $2-1$ of Algorithm 2. Since the difference of their summations in the above cases cannot exceed 1 , $\left|\sum_{p \in M_{1}} a_{p q}-\sum_{p \in M_{2}} a_{p q}\right| \leq 1$ holds. 
(iii-2) $\{-1,-1,-1\}$ : By case 2-3 of Algorithm 2, all rows are partitioned to different partitions $\left\{p, p^{\prime}\right.$ (or $\left.\left.p^{\prime \prime}\right)\right\}$ and $\left\{p^{\prime \prime}\left(\right.\right.$ or $\left.\left.p^{\prime}\right)\right\}$. Thus, $\left|\sum_{p \in M_{1}} a_{p q}-\sum_{p \in M_{2}} a_{p q}\right| \leq 1$ holds.

(iv) $N_{q}=4$ : Note that we have only one +1 and three -1 among $a_{p q}$ for $p \in M$. By case $2-2,\{1,-1\}$ is first assigned to the same partition, then $\{-1,-1\}$ is divided into $M_{1}$ or $M_{2}$. Since its summation is zero, $\left|\sum_{p \in M_{1}} a_{p q}-\sum_{p \in M_{2}} a_{p q}\right| \leq 1$ holds.

For all possible cases, we prove that the partition satisfies condition (ii) of Theorem 1. Thus, the constraint matrix $A$ is totally unimodular.

Therefore, we have the solution of the original integer problem given in Equation 9 in polynomial time.

\section{Competing interests}

The authors declare that they have no competing interests.

\section{Acknowledgements}

This work was supported by the National Research Foundation of Korea (NRF) Grant funded by the Korean Government (MSIP) (No. 2012R1A1A1014755, NRF-2013R1A1A1006823) and Korea Naval Institute for Ocean Research.

\section{Author details}

${ }^{1}$ Department of Computer Science, Naval Academy, Changwon 645-797, South Korea. ${ }^{2}$ Department of Software Design \& Management, Gachon University, Seongnam 461-701, South Korea. ${ }^{3}$ School of Computer Science and Engineering, Seoul National University, Seoul 151-744, South Korea.

Received: 30 January 2014 Accepted: 3 November 2014 Published: 17 November 2014

\section{References}

1. J Ott, D Kutscher, in Proceedings of The 23rd Annual Joint Conference of the IEEE Computer and Communications Societies (INFOCOM'04). Drive-thru Internet: IEEE 802.11 b for 'automobile' user, (7-11 March 2004)

2. D Hadaller, S Keshav, T Brecht, S Agarwal, in Proceedings of The 5th International Conference on Mobile Systems, Applications, and Services (MobiSys'07). Vehicular opportunistic communication under the microscope (San Juan, Puerto, Rico, 11-14 June 2007)

3. V Bychkovsky, B Hull, AK Miu, H Balakrishnan, S Madden, in Proceeding of The 12th Annual International Conference on Mobile Computing and Networking (MobiCom'06). A measurement study of vehicular internet access using in situ Wi-Fi networks (Los Angeles, CA, 24-29 September 2006)

4. A Giannoulis, M Fiore, Knightly E W, in Proceedings of The Sixth International Conference on Mobile Systems, Applications, and Services (MobiSys'08). Supporting vehicular mobility in urban multi-hop wireless networks (Breckenridge, Colorado, 17-20 June 2008)

5. A Balasubramanian, R Mahajan, A Venkataramani, B Levine, J Zahorjan, in Proceeding of ACM SIGCOMM. ViFi: interactive WiFi connectivity for moving vehicles (Seattle, WA, 17-22 August 2008)

6. J Eriksson, H Balakrishnan, S Madden, in Proceeding of The 14th Annual International Conference on Mobile Computing and Networking (MobiCom'08). Cabernet: vehicular content delivery using WiFi (San Francisco, CA, 14-19 September 2008)

7. N Banerjee, MD Corner, D Towsley, BN Levine, in Proceeding of The 14th Annual International Conference on Mobile Computing and Networking (MobiCom'08). Relays, base stations, and meshes: enhancing mobile networks with infrastructure (San Francisco, CA, 14-9 September 2008)

8. K Kim, Y Kim, S Pack, N Choi, A SNR-based admission control scheme in WiFi-based vehicular networks. EURASIP J. Wireless Commun. Netw. 2011(204) (2011)
9. P Deshpande, A Kashyap, C Sung, SR Das, in Proceeding of ACM MobiSys'09. Predictive methods for improved vehicular Wi-Fi access (Krakao, Poland, 22-25 June 2009)

10. BG Lee, Choi S, Broadband Wireless, Access \& Local Networks: Mobile WiMAX and Wi-Fi, (Artech House, Norwood, 2008), pp. 935-982

11. M Kim, Z Liu, S Parthasarathy, D Pendarakis, H Yang, in Proceeding of The 27th IEEE Conference on Computer Communications (INFOCOM'08). Association control in mobile wireless networks (Phoenix, AZ, 13-18 April 2008)

12. L Xie, Q Li, W Mao, J Wu, D Chen, in Proceeding of The 17th IEEE International Conference on Network Protocols (ICNP'09). Achieving efficiency and fairness for association control in vehicular networks (Princeton, New Jersey, 13-16 October 2009)

13. TOPIS. http://topis.seoul.go.kr/. Accessed 10 Feb 2011

14. Y Kim, K Kim, S Pack, W Lee, Analysis of session handover probability in NEMO-based vehicular networks. Springer Wireless Personal Commun 61(4), 697-710 (2011)

15. J-H Lee, T Ernst, Lightweight network mobility within PMIPv6 for transportation systems. IEEE Syst. J. 5(3), 352-361 (2011)

16. J-H Lee, T Ernst, N Chilamkurti, Performance analysis of PMIPv6 based Network Mobility for intelligent transportation systems. IEEE Trans. Vehicular Technol. 61(1), 74-85 (2012)

17. J Yoo, B Choi, M Gerla, in Proceedings of IEEE International Conference on Network Protocols (ICNP'10). An opportunistic relay protocol for vehicular road-side access with fading channels (Kyoto, Japan, 5-8 October 2010)

18. K Kim, AW Min, D Gupta, P Mohapatra, J Singh, in Proceeding of The 30th IEEE International Conference on Computer Communications (INFOCOM'11). Improving energy efficiency of Wi-Fi sensing on smartphones (Shanghai, China, 10-15 April 2011)

19. Wigle. http://wigle.net. Accessed 10 Feb 2011

20. J Pang, B Greenstein, D McCoy, M Kaminsky, S Seshan, in Proceedings of The 7th International Conference on Mobile Systems, Applications, and Services (MobiSys'09). Wifi-Reports: improving wireless network selection with collaboration (Krakao, Poland, 22-25 June 2009)

21. GL Nemhauser, LA Wolsey, Integer and Combinatorial Optimization. (Wiley, New York, 1988), pp. 535-607

22. LA Wolsey, Integer Programming. (Wiley, New York, 1998), pp. 37-52

23. H Lee, S Kim, O Lee, S Choi, S Lee, in Proceeding of The 11-th ACM International Conference on Modeling, Analysis and Simulation of Wireless and Mobile Systems (MSWiM'08). Available bandwidth-based association in IEEE 802.11 wireless LANs (Vancouver, Canada, 27-31 October 2008)

24. J Hwang, H Lee, J Choi, J Yoo, C Kim, An efficient association control method in vehicular network with mobile hotspots. KSII Trans. Internet Inf. Syst. (TIIS). 5(5), 888-908 (2011)

25. Olleh WiFi, http://zone.wifi.olleh.com/. Accessed 10 Feb 2011

26. GAMS. http://www.gams.com/. Accessed 10 Feb 2011

doi:10.1186/1687-1499-2014-192

Cite this article as: Hwang et al:: A theoretical approach to optimal association control in vehicular Wi-Fi networks. EURASIP Journal on Wireless Communications and Networking 2014 2014:192.

\section{Submit your manuscript to a SpringerOpen ${ }^{\odot}$ journal and benefit from:}

- Convenient online submission

- Rigorous peer review

- Immediate publication on acceptance

- Open access: articles freely available online

- High visibility within the field

- Retaining the copyright to your article

Submit your next manuscript at $>$ springeropen.com 